\title{
Regularization for FWI with Geological Incoherence Index
}

Raphael Vieira Menezes de Souza, Franciane Conceição Peters, Webe João Mansur, Luiz Alberto Santos and Kristian David Torres Bautista

Copyright 2019, SBGf - Sociedade Brasileira de Geofísica

This paper was prepared for presentation during the $16^{\text {th }}$ International Congress of the Brazilian Geophysical Society held in Rio de Janeiro, Brazil, 19-22 August 2019.

Contents of this paper were reviewed by the Technical Committee of the $16^{\text {th }}$ International Congress of the Brazilian Geophysical Society and do not necessarily represent any position of the SBGf, its officers or members. Electronic reproduction or storage of any part of this paper for commercial purposes without the written consent of the Brazilian Geophysical Society is prohibited.

\section{Abstract}

The present work advances in the use of the Full Waveform Inversion (FWI) for generation of images based on the complete wave equation. In particular, the use of the Geological Inconsistency Index - Gll was analyzed as a constraint in the process of regularization of the inverse problem. Computational experiments presented in this work show that the use of Gll leads the solution to a more likely solution, with geological meaning providing more realistic images of the subsurface compared to the classic FWI and the FWI with Total Variation Regularization.

\section{Introduction}

The Full Waveform Inversion (FWI) is a well-established technique that uses the complete wave equation to estimate acoustic subsurface parameters, providing highresolution images. The first work that introduced the FWI concept was developed by Lailly and Bednar (1983) followed by Tarantola (1984) in the beginning of the 1980s. In these works, the authors developed the classical FWI formulation, seeking to solve the inverse problem in the time domain. While many theories concerning FWI have been developed from the 1980s to 1990s, large-scale FWI employment in the oil and gas industry has only been put into the production line in the last 20 years.

In general terms, the objective of a FWI algorithm is to find the best values of properties that, using wave propagation modeling, generate a synthetic seismogram close to the seismogram recorded in the survey. However, it is known from the literature that FWI is an illposed problem, which fails in the criteria of uniqueness and solution stability (Aster et al., 2005; Fichtner, 2010; Virieux and Operto, 2009).

To stabilize the process of solving ill-posed problems, current works have been developed in the FWI area using regularization (Dai et al., 2017; Maharramov et al., 2016; Asnaashari et al., 2013), and it is in this context that the present work was developed. We use the Geological Incoherence Index (GII), proposed by Santos (2012), as a constraint for the inverse problem.
The use of GII in the regularization of the inverse problem seeks to encompass geological features, which are neglected in conventional FWI. Gll regularization makes the problem less ill-posed and leads to more stable solutions.

\section{Method}

The FWI imaging problem is a data fit problem in which an initial model of parameters $\chi$ is iteratively updated using some optimization process and converges to a model hopefully close to the geological model of the subsurface of the area where the seismic survey was performed to provide the observed data.

It means that the goal is to find a parameter vector $\chi^{*}$ that minimizes the functional $\boldsymbol{F}(\chi)$, given by the difference between the seismic data observed in the field and the data obtained with the computational simulation.

In the present work, the Finite Difference Method (FDM) is used to obtain the numerical solution of the forward problem, the computacional of synthetic seismograms. The wave propagation is modeled by a generalization of the Helmholtz equation, given by:

$$
\frac{\omega}{\kappa(x)} u(x, \omega)+\nabla \cdot\left(\frac{1}{\rho} \nabla u(x, \omega)\right)=s(x, \omega),
$$

where $u(x, \omega)$ and $s(x, \omega)$ are the components of the pressure field and source term respectively, in position $x$ and in frequency $\omega, \kappa$ is the stiffness coefficient and $\rho$ is the density.

To solve the inverse problem, in this paper, the technique known as multiscale is used. The use of this strategy assumes that the objective function for lower frequencies is smoother and consequently has few local minima. In this approach, the seismic data is divided into frequency bands, and the inversion process is used to update the model for each band, in ascending magnituder of frequencies.

For the minimization of the objective function, we use the L-BFGS method, which is a limited memory version of the BFGS method, and has been applied to solve problems with a large number of variables, such as FWI, (Nocedal and Wright, 2006).

To stabilize the inversion process, we propose the use of Gll a regularization technique for the inverse problem. The Gll was proposed by Santos (2012) and is used to 
quantify the consistency between the interfaces of the layers and the velocity gradient of the model. To compute the Gll it is necessary to define the velocity gradient vector:

$$
\nabla v=\left(\frac{\partial v}{\partial x}, \frac{\partial v}{\partial z}\right)
$$

and the angle $\theta_{v}$ that the gradient vector makes with the horizontal direction at each node of the numerical model:

$$
\theta_{v}=\arctan \left(\frac{\frac{\partial v}{\partial z}}{\frac{\partial v}{\partial x}}\right)
$$

If we consider all points of the domain, it is possible to compute the field $\theta_{v}$. In addition, it is necessary to calculate the angle $\theta_{s}$ between the normal vector at each point of the interface and the horizontal. Once these angles are calculated, their values are extrapolated along their respective layers. Thus, at the end of the process the field $\theta_{s}$ is supposed to be very similar to the field $\theta_{v}$, for a model with low Gll.

From these premises, Santos (2012) has defined the GIl as being:

$$
\boldsymbol{G I I}=\frac{\sum_{i=1}^{n}\left|\theta_{v_{i}}-\theta_{S_{-} i}\right|}{n},
$$

where $n$ is the number of nodes of the numerical model.

The proposal is to incorporate the Gll into the functional as a regularization term, as follows:

$$
\boldsymbol{f}(\chi)=\|\left.\boldsymbol{F}(\chi)\right|_{2} ^{2}+\alpha_{n} \boldsymbol{G I I}
$$

where $\alpha_{n}$ is a regularization parameter.

Another type of regularization well known in the literature, and that will be used in this work to generate reference results, is the Total Variation Regularization (TV). The main advantage of the TV Regularization is its ability of reducing noise by preserving important features in an image, like interfaces (Alemie, 2017). Rudin et al. (1992) point out that TV regularization consists of a change in the objective function that can be described as:

$$
\boldsymbol{f}(\chi)=\left.|| \boldsymbol{F}(\chi)\right|_{2} ^{2}+\alpha|| \chi \|_{T V^{\prime}}
$$

where the term corresponding to the TV regularization is defined by:

$$
\left.|| \chi\right|_{T V}=\sum_{i=1}^{N x} \sum_{j=1}^{N z} \sqrt{\left|\left(\nabla_{\mathrm{x}} \chi\right)_{i, j}\right|^{2}+\left|\left(\nabla_{z} \chi\right)_{i, j}\right|^{2}+\epsilon}
$$

where $\left(\nabla_{\mathrm{x}} \chi\right)_{i, j}=\chi_{i+1, j}-\chi_{i, j},\left(\nabla_{\mathrm{z}} \chi\right)_{i, j}=\chi_{i, j+1}-\chi_{i, j}$ and $\epsilon$ is a constant added to ensure that $\|\chi\|_{T V}$ is differentiable.

Results

To demonstrate the performance of the proposed methodology, two velocity models are used in the experiments: the modified Amoco model and the modified Marmousi model. All models are discretized with a regular mesh of finite differences with spacing of 12 meters with $767 \times 293$ nodes.

The observed data to be inverted were generated synthetically using 188 sources spaced uniformly by a distance of $48 \mathrm{~m}$ between each of them. In addition, 382 receivers were spaced by a distance of $24 \mathrm{~m}$ from each other. The non-reflexive boundaries are implemented by a combination of the strategies presented by Reynolds (1978) and Berenger (1994).

To simulate the presence of noise in the observed data, up to $30 \%$ of Gaussian noise was inserted in the seismograms used in the experiments.

For the inversion, the frequencies $6 \mathrm{~Hz}, 8 \mathrm{~Hz}, 10 \mathrm{~Hz}, 12$ $\mathrm{Hz}$ and $14 \mathrm{~Hz}$ were used, and the stop criterion for each frequency was the maximum number of iterations (10 iteration per frequency) or 10 consecutive fails in reduction of the objective function at the same iteration.

The relative error used to evaluate the results is defined as:

$$
E=\frac{1}{n_{x} n_{z}} \sum_{i=1}^{n_{x}} \sum_{j=1}^{n_{z}}\left|\chi_{i, j}-\overline{\chi_{\imath, j}}\right|
$$

where $\chi_{i, j}$ and $\overline{\chi_{i, j}}$ are the velocity values at the node $(i, j)$ of the inverted and exact models, respectively.

We also present comparative results of what we define as vertical profile average error, in which the differences between the velocities of the recovered model and the exact model for each one of the 767 vertical profiles of the model are presented.

Figure 1 presents exact models (Amoco and Marmousi), initial velocity models, inversion results obtained with Gll Regularization and vertical profiles of average error and the relative error.

As can be seen in Figures $1(\mathrm{~g})$ and $1(\mathrm{~h})$, the results obtained with the use of Gll regularization presented smaller errors, compared to FWI without regularization and FWI with Total Variation Regularization. This improvement in the results with the use of GII is more 
(a)
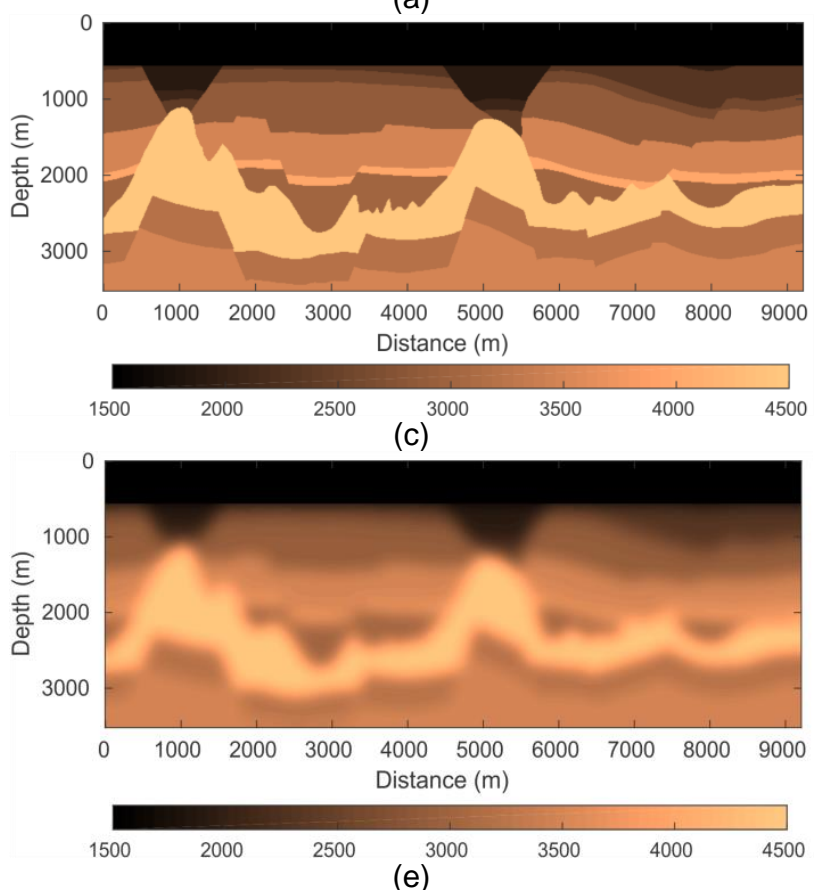

(e)

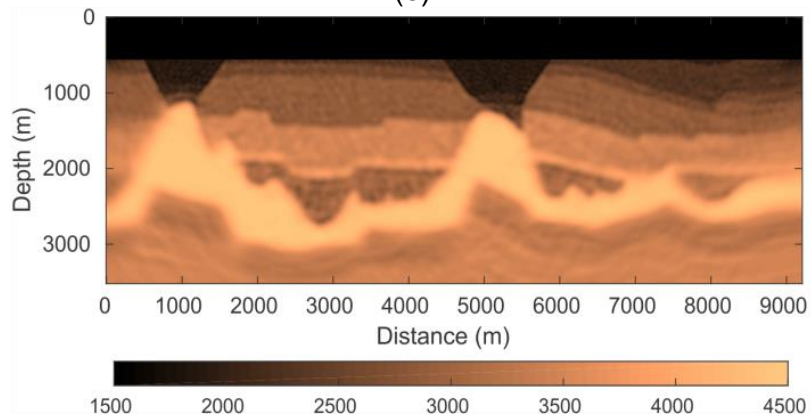

(g)

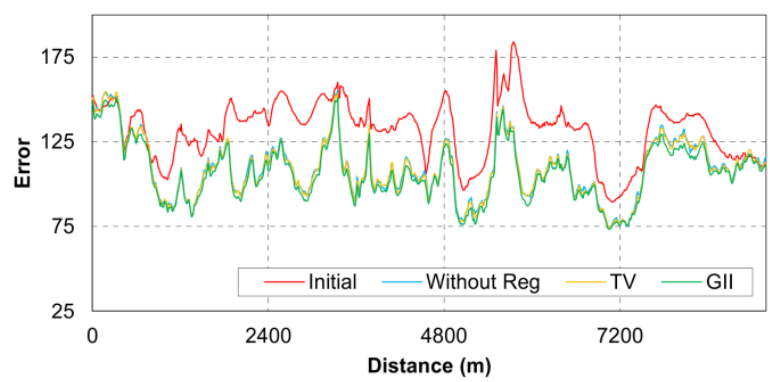

(i)

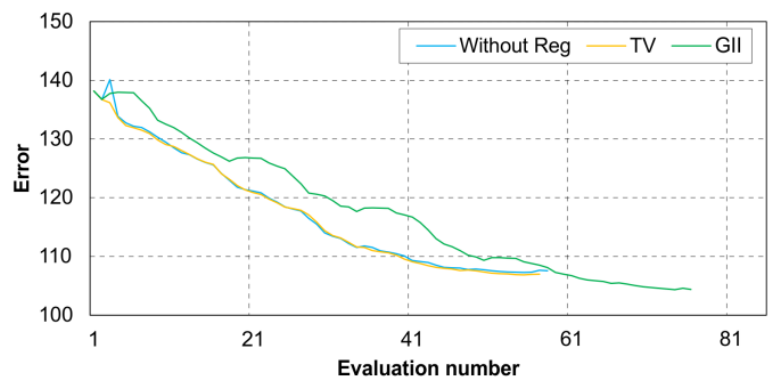

(b)
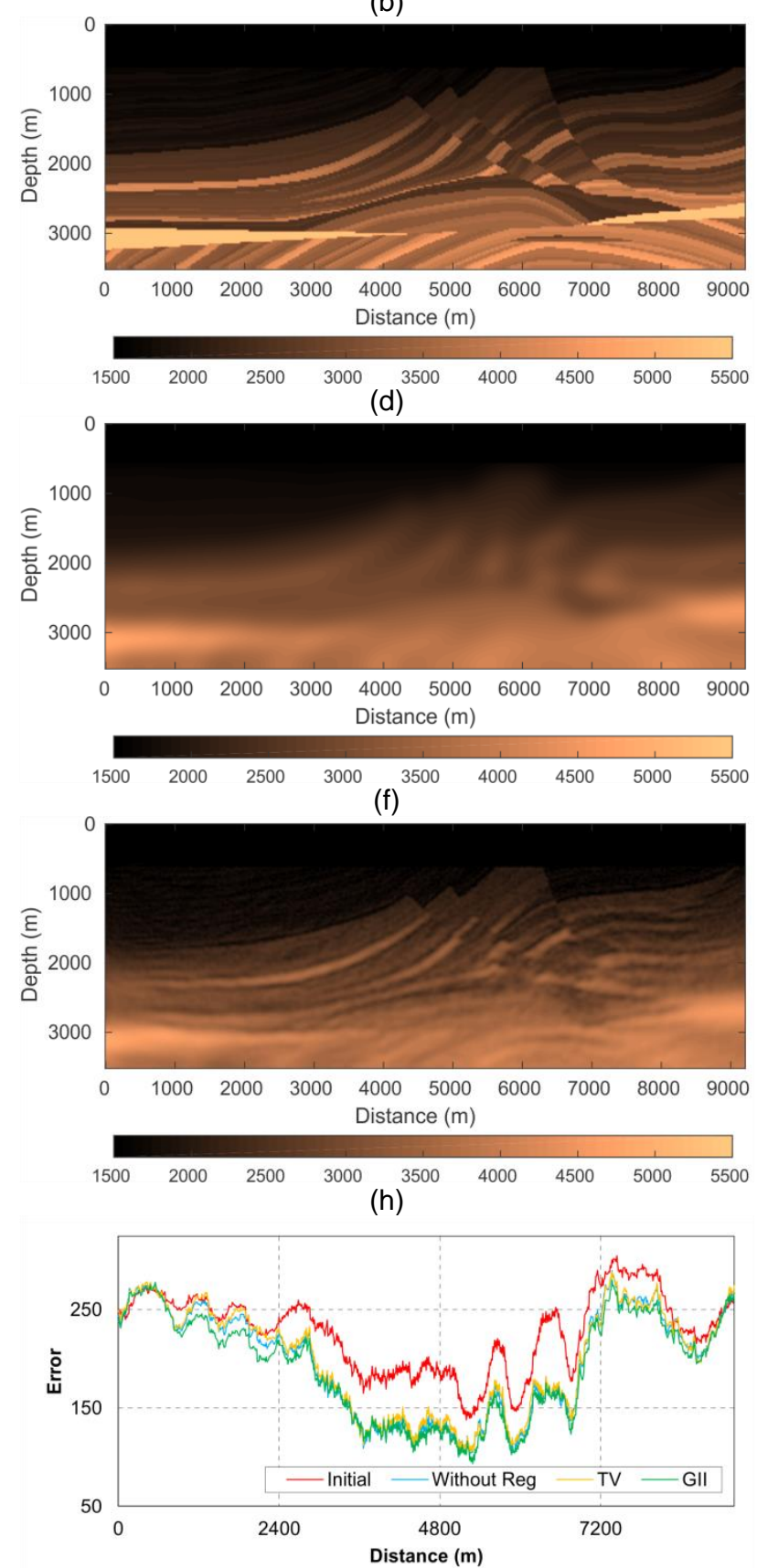

(j)

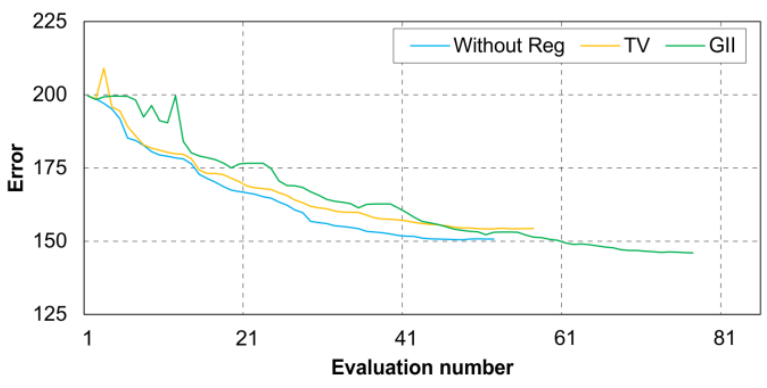

Figura 1 - Exact models $(a, b)$, initial velocity models $(c, d)$, inversion results obtained with Gll Regularization (e,f), vertical profile average error $(g, h)$ and vertical profile relative errors $(I, j)$. 
noticeable mainly in the regions farthest from the center of the model, with low illumination.

Figures $1(\mathrm{l}, \mathrm{j})$ show the relative errors of the models during the objective function evaluations in the inversion. It is noted that the use of Gll as a regularization term presents better results than the other methodologies presented here, but the computational cost of this approach is somewhat higher, because Gll regularization requires more evaluations of the objective function during the inversion.

\section{Conclusions}

In this work, we evaluated the use of the Geological Incoherence Index - GII, proposed by Santos (2012), as a FWI regularization, in order to make the problem less illposed and more stable. The use of this methodology sought to encompass geological characteristics, which are neglected in conventional FWI.

Although Gll regularization requires more evaluations of the objective function during the inversion process, the use of Gll has presented better results than the other strategies in the numerical experiments performed with Amoco and Marmousi models.

\section{Acknowledgments}

This study was financed in part by the Coordenação de Aperfeiçoamento de Pessoal de Nível Superior-Brasil (CAPES) - Finance Code 001, CNPq (306.933/2014-4, 423.794/2016-7, 306933/2014-4, 130.811/2017-3, 306191/2018-0, 423114/2018-2), Faperj (203.234/2016, 210.210/2016, 201.453/2014, 203.021/2017, 200.262/2015) and Petrobras (CENPES - 014987).

\section{References}

ALEMIE, W.M. Time-Lapse Full Waveform Inversion Methods.Ph.D. thesis; University of Alberta; 2017.

ASNAASHARI, A., BROSSIER, R., GARAMBOIS, S., AUDEBERT, F., THORE, P., VIRIEUX, J. Regularized seismic full waveform inversion with prior model information. Geophysics 2013.

ASTER, R., THURBER, C., BORCHERS, B. Parameter estimation and inverse problems. New York: Academic Press, 2005.

BERENGER, J. A perfectly matched layer for the absorption of electromagnetic waves. Journal of computational physics 1994;114(2):185-200.

DAI, M.X., CHEN, J.B., CAO, J.. I1-regularized fullwaveform inversion with prior model information based on orthant-wise limited memory quasi-newton method. Journal of Applied Geophysics 2017.

FICHTNER, A.. Full seismic waveform modelling and inversion. Springer Science \& Business Media, 2010.
LAILLY, P., BEDNAR, J.. The seismic inverse problem as a sequence of before stack migrations. In: Conference on inverse scattering: theory and application. Siam Philadelphia, PA; 1983. p. 206-220.

MAHARRAMOV, M., BIONDI, B., MEADOWS, M. Simultaneous tv regularized time-lapse fwi with application to field data. In: 78th EAGE Conference and Exhibition 2016-Workshops. 2016.

NOCEDAL, J., WRIGHT, S.. Numerical optimization. Springer Science \& Business Media, 2006.

REYNOLDS, A.C.. Boundary conditions for the numerical solution of wave propagation problems. Geophysics 1978;43(6):1099-1110.

RUDIN, L.I., OSHER, S., FATEMI, E.. Nonlinear total variation based noise removal algorithms. Physica $D$ : Nonlinear Phenomena 1992;60(1):259-268.

SANTOS, L.A.. Inversão tomográfica sequencial para o campo de velocidades sísmicas baseada em difrações e critérios geológicos. Ph.D. thesis; COPPE/UFRJ, Rio de Janeiro, RJ, Brasil; 2012.

TARANTOLA, A. Inversion of seismic reection data in the acoustic approximation. Geophysics 1984;49(8):12591266.

VIRIEUX, J., OPERTO, S. An overview of full-waveform inversion in exploration geophysics. Geophysics 2009;74(6):WCC1-WCC26. 